\title{
Performance Analysis of Reactive Shortest Path and Multi-path Routing Mechanism With Load Balance
}

\author{
Peter P. Pham and Sylvie Perreau \\ Institute for Telecommunications Research \\ University of South Australia \\ Mawson Lakes SA 5095 Australia \\ Phone: 61-83025188. Fax: 61-83023873 \\ Email: \{ppham,sylvie\}@ spri.levels.unisa.edu.au
}

\begin{abstract}
Research on multi-path routing protocols to provide improved throughput and route resilience as compared with single-path routing has been explored in details in the context of wired networks. However, multi-path routing mechanism has not been explored thoroughly in the domain of ad hoc networks. In this paper, we analyze and compare reactive single-path and multi-path routing with load balance mechanisms in ad hoc networks, in terms of overhead, traffic distribution and connection throughput. The results reveals that in comparison with general single-path routing protocol, multi-path routing mechanism creates more overheads but provides better performance in congestion and capacity provided that the route length is within a certain upper bound which is derivable. The analytical results are further confirmed by simulation.
\end{abstract}

Index Terms - ad-hoc networks, load balancing, multi-path routing, overheads.

\section{INTRODUCTION}

$\mathbf{M}$ OBILE Ad Hoc Networks (MANETs) are collections of wireless mobile nodes, constructed dynamically without the use of any existing network infrastructure or centralized administration. Due to the limited transmission range of wireless network interfaces, multiple hops may be needed for one node to exchange data with another one across the network. MANETs are characterized by limited power resource, high mobility and limited bandwidth. Routing in MANETs can be accomplished through either single path or multiple paths. When using single-path routing protocols, the traffic is distributed through one route and is therefore less flexible than in multi-path routing protocols. The problem of two entities communicating using multiple paths has been considered widely in various contexts for wired networks [1], [2], [3], [4], [5]. It was shown that multi-path routing mechanism provides better throughput than single-path routing protocols [2], [3]. Although research on multi-path routing protocols has been covered quite thoroughly in wired networks, similar research for wireless networks is still in its infancy. Some multi-path routing protocols for MANETs have been proposed in [6], [7], [8], [9]. However, the performance of these protocols are only assessed by simulations in certain limited scenario. Although some recent papers provide analytical models for multi-path routing
[10], [11], they are limited on a single aspect of multi-path routing such as route discovery frequency or error recovery. To the best of our knowledge, there has been no paper which provides an analytical model which allows comparing the performance of reactive shortest single-path routing and multi-path routing with load balance. In this paper, we propose models to analyze and compare reactive single-path and multi-path routing protocols in terms of overheads, traffic distribution and connection throughput. Thereafter, the terms "single-path routing" and "multi-path routing" are equivalent to "shortest single-path routing" and "multi-path routing with load balance" respectively. In addition, we focus our analysis only on reactive routing mechanism. The overhead analysis in this paper is only applicable for reactive routing mechanism. However, the results regarding the traffic distribution and connection throughput is applicable for both proactive and hybrid routing mechanisms. The outcome from analytical models is further validated by simulation. The remaining of this paper is organized as follows. Section II provides general information on reactive routing mechanism. Section III gives a detailed analysis of overhead for both single-path and multi-path routing techniques. In section IV, we analyze the traffic distribution for both mechanisms and section $\mathrm{V}$ concentrates on the capacity analysis. We finally conclude this study discuss future research directions in section VI.

\section{Reactive Routing Mechanism}

Reactive routing protocols in MANETs consist of the following dominant candidates Dynamic Source Routing (DSR) [12], Adhoc On-demand Distance Vector Routing (AODV) [13] and Temporally Ordered Routing Algorithm (TORA) [14]. They all have two main phases in common: Route Discovery and Route Maintenance.

\section{A. Route Discovery}

In this phase, the source node $\mathrm{S}$ broadcasts a route request packet (RRQ) to locate the destination node $\mathrm{D}$ in the network. The first node receiving the RRQ that has a valid route for node D initiates a route reply packet (RRP) back to node $S$ containing a list of nodes a long the path from node $\mathrm{S}$ to node $\mathrm{D}$. 


\section{B. Route Maintenance}

The Route Maintenance phase ensures that the paths stored in the Route Cache are valid. If the data link layer of a node detects a transmission error, the node creates a route error packet (ERR) and transmits it to the source. For error detection, several acknowledgement mechanisms may be used such as ACK packet for each successfully-transmitted packet, link detection mechanism in $802.11 \ldots$ When receiving ERRs, the sources check their route caches and delete the routes containing the failed links. They can either attempt to use other alternate routes in their caches when using multi-path routing mechanism or invoke another route discovery when using single-path routing mechanism.

\section{OVERHEADS ANALYSIS}

\section{A. Route Creation Frequency}

Let us firstly review the results of [11]. This significant result indicates that the route creation rate for multi-path routing strategy is lower than it is for single-path routing. The link's lifetime is assumed to be independent and identically distributed (iid) exponential random variables with mean $l$. Since a route fails when any links in its path breaks, the lifetime of a route with $L$ links is also an exponentially distributed random variable with a mean of $l / L$.

Theorem 1: Denoting by $\mu_{i}=l / L_{i}$, The probability density function (pdf) of $T$, the time between successive route discoveries, is given by:

$$
f_{T}(t)=\prod_{i=1}^{N}\left(1-\exp \left(-\mu_{i} t\right)\right) \sum_{i=1}^{N} \mu_{i} \frac{\exp \left(-\mu_{i} t\right)}{1-\exp \left(-\mu_{i} t\right)}
$$

Comment: The expected value of $\mathrm{T}$ can be derived by knowing the hop-wise lengths of all the routes $k i, i=1, \ldots, N$. It was also shown in [11] that using multi-path routing can achieve $25 \%$ reduction in route discoveries rate for 3-4 hops routes as compared with single-path routing. This reduction is because in multi-path routing, route discovery is only initiated when all the routes to the destination are broken whereas in single-path routing, it is done when one single route is broken.

\section{B. Overheads Analysis by Intuition}

Overheads in reactive routing protocols are caused in the following phases: Route Discovery, Route Maintenance, and Data Transmission. The overheads for single-path and multi-path routing mechanisms are analyzed according to these phases.

1) Route Discovery: Route Discoveries for single-path and multi-path routing mechanisms are shown in Fig 1 and Fig 2 respectively. Clearly shown, the number of broadcasted RRQs is the same for both single-path and multi-path routing. However, when the destination sends the RRPs back to the source, because it has to send $N_{u}$ ( $N_{u}$ is the number of multiple paths created in the Route Discovery phase) RRQs to correspond to $N_{u}$ RRQs, the overheads of multi-path routing in Route Discovery phase is higher than that of single-path routing. The extra overhead is proportional the number of paths $N u$.
2) Route Maintenance: In this phase, when a link is broken, an Error Packet (ERR) is sent back to the source to indicate the route breakage. In multi-path routing, since there are multiple paths for each source-destination pair, assuming the probability of link breakage and the route length for all the routes are the same, the number of route breakages is proportional to the number of paths. Therefore, it can be deduced that in multipath routing, the number of ERRs is higher than in single-path routing, i.e. more overheads.

3) Data Transmission: During this stage, overhead is mainly due to the overhead portion of the data packets which is dependent on the routing protocols themselves. For some protocols such as DSR, the complete route from the source to the destination is stored inside the overhead portion of the data packets. However, in other ones such as AODV, only next node is stored in the data packet which results in less overhead as compared with DSR.

4) Comment: In summary, we can clearly see that there is a trade-off between single-path and multi-path routing mechanisms. In multi-path routing, overheads in multi-path routing are high due to extra RRPs and ERRs. However, the frequency of route discoveries in multi-path routing is lower than in single-path routing as claimed in [11]. Hence, an analytical model is established in the following section to get a better understanding of the trade-off.

\section{Overhead Analysis Using Analytical Model}

1) Network Model: We assume that mobile nodes are distributed uniformly with node density $\delta$ inside a disk of radius $R$. We also assume that there are $N$ nodes in the network. $N$ is related to the node density and the disk radius by the following expression $N=\pi R^{2} \delta$. Each link has a link breakage rate of $\mu$, i.e. a link has a average lifetime of $1 / \mu$ seconds on average. Furthermore, we assume that the average route length (in terms of number of hops) for single-path routing is $L_{s}$ and for multipath routing is $L_{m}$. Since single-path routing mechanism uses shortest routes, we obviously have $L_{m}>L_{s}$. In addition, $L_{e}$ is assumed to be the average length of the route from the source to the node where a link breakage occurs. For multi-path routing, $N_{u}$ represents the number of paths for each source-destination pair. In addition, the number of active connections per node is denoted by $A_{c}$ for both routing mechanisms. Furthermore, the size of RRQ, RRP and ERR are respectively denoted as $M_{r q}$, $M_{r p}, M_{e}$ respectively. Finally, a route discovery takes $T$ seconds to find the routes to the destination. All the parameters are summarized in Table I:

2) Overhead due to RRQs:

- Single-path Routing Mechanism:

Assuming that $N$ nodes each broadcast a RRQ $\lambda_{s}$ times per second, the total overhead created by RRQs is obviously $M_{r q} \lambda_{s} N^{2}$. $\lambda_{s}$ (i.e the route discovery frequency) is related to link breakage as $\lambda_{s}=\mu L_{s}$. Hence, the amount of overheads due to the RRQs is $M_{r q} \mu L_{s} N^{2}$.

- Multi-path Routing Mechanism:

Using a similar argument as above, the amount of overheads due to RRQs is $M_{r q} \lambda_{m} N^{2}$ where $\lambda_{m}$ is the frequency of route discovery for multi-path routing algo- 
TABLE I

SUMMARY OF PARAMETERS

\begin{tabular}{|l|l|}
\hline$N$ & Number of nodes \\
\hline$N_{u}$ & Number of routes per source-destination pair \\
\hline$L_{e}$ & Average length of error route \\
\hline$\mu$ & Link breakage rate \\
\hline$L_{s}$ & Average length of a route for single-path routing. \\
\hline$L_{m}$ & $\begin{array}{l}\text { Average length of a route for multi-path routing } \\
\text { mechanism. }\end{array}$ \\
\hline$A_{c}$ & Number of active routes per node \\
\hline$M_{r q}$ & Size of the request packet \\
\hline$M_{e}$ & Size of error request packet \\
\hline$M_{r p}$ & Size of reply packet \\
\hline$\epsilon$ & Inter-arrival rate \\
\hline$P$ & Overhead portion of a data packet. \\
\hline$M_{d}$ & Size of the data packet \\
\hline$T$ & Average delay for route creation \\
\hline$\lambda_{s}$ & $\begin{array}{l}\text { Route discovery frequency for single-path rout- } \\
\text { ing }\end{array}$ \\
\hline$\lambda_{m}$ & Route discovery frequency for multi-path routing \\
\hline
\end{tabular}

rithm. This parameter can be calculated using Theorem 1 .

3) Overhead due to RRPs:

- Single-path Routing Mechanism:

Reply packets follow $L_{s}$ hops to return back to the source. Since the rate of sending the RRPs is the same as the rate of sending RRQs, the overhead created by the RRPs, is $M_{r p} \mu L_{s}^{2} N$.

- Multi-path Routing Mechanism:

Since the destination node replies to $N_{u}$ RRQs, the overhead due to RRPs is $M_{r p} \lambda_{m} L_{m} N N_{u}$. Note that the fact that $\lambda_{m}$ is smaller than $\lambda_{s}$ balances the fact that the number of RRPs are increased by a factor of $N_{u}$ compared to single-path routing.

4) Overheads due to ERRs: When a link is broken, an Error Packet is sent back to the source to signal the link breakage. Recall that $L_{e}$ is the average length of the path from the broken link to the source $\left(L_{e}<L_{s}<L_{m}\right)$. Since the error packet has to travel $L_{e}$ links to the source, this effectively produces $L_{e}$ error packets per route broken.

- Single-path Routing Mechanism:

Since the link breakage rate is $\mu$, the route breakage rate for a route with $L_{s}$ links is $\mu L_{s}$. For each node, the average number of active routes is $A_{c}$. Therefore, for a node, the route breakage rate is $\mu L_{s} A_{c}$. Therefore, in a $N$-node network, the average number of overheads due to error packets is $\mu L_{s} A_{c} N L_{e} M_{e}$.

- Multi-path Routing Mechanism:

In multi-path routing, since each source-destination pair maintains $N_{u}$ routes, the overheads due to error packets is $N_{u} \mu L_{m} L_{e} A_{c} N M_{e}$.

5) Overheads Due to Data Transmission: The overheads created during data transmission is due to the overhead portion of data packets. We assume that the each route discovery is accomplished in $\mathrm{T}$ seconds on average. Furthermore, each mobile node is a simple source with data transmission rate of $\epsilon$ once the route discovery is completed.

- Single-path Routing Mechanism:

Since the route discovery rate is $\lambda_{s}$, the interval between each route discoveries is on average $1 / \lambda_{s}$. Each route discovery takes on average $T$ seconds. Therefore, the actual time for data transmission is $\left(1 / \lambda_{s}-T\right)$ seconds. The number of data packets sent during that interval is $\left(1 / \lambda_{s}-\right.$ $T) \epsilon$. Thus, data packets are sent with an average rate of $\lambda_{s} \epsilon\left(1 / \lambda_{s}-T\right)$ packets/sec. Since each data packet has to travel $L_{s}$ hops to the destination, the total amount of overhead is $\lambda_{s} \epsilon\left(1 / \lambda_{s}-T\right) P L_{s}=\mu L_{s} \epsilon\left(1 /\left(\mu L_{s}\right)-T\right) P L_{s}$.

- Multi-path Routing Mechanism:

Using a similar derivation as above, the total amount of overheads for multi-path routing is $\lambda_{m} \epsilon\left(1 / \lambda_{m}-T\right) P L_{m}$ where $\lambda_{m}$ can be calculated using Theorem 1. (we do not include the derivation of this calculation in this paper by lack of space).

6) Summary: The total amount of overheads due to RRQs, RRPs, ERRs and data packets for single-path and multi-path respectively denoted by $O v_{s}$ and $O v_{m}$ can be expressed as:

$$
\begin{aligned}
& O v_{s}=M_{r q} \lambda_{s} N^{2}+M_{r p} \lambda_{s} L_{s} N+ \\
& \quad \mu L_{e} L_{s} A_{c} N M_{e}+\mu L_{s} \epsilon\left(1 /\left(\lambda_{s}-T\right) P L_{s}\right. \\
& O v_{m}=M_{r q} \lambda_{m} N^{2}+M_{r p} \lambda_{m} N L_{m} N_{u} \\
& \quad+\mu L_{e} L_{m} A_{c} N M_{e} N_{u}+\mu \epsilon\left(1 / \lambda_{m}-T\right) P L_{m}
\end{aligned}
$$

In Fig 3, we have plotted $O v_{s}$ and $O_{m}$ as functions of the number of paths $N_{u}$. One can see that there is no significant increase in overheads for $N_{u}$ up to 3 . This confirms the fact that in the literature, authors often mentioned that $N_{u}=3$ provides an optimum trade off [11], [3]. This claim is usually based on simulation results and the study provided in this paper confirms this observation. In Fig $4, N_{u}=3$ and $O v_{s}$ and $O v_{m}$ are compared as the link breakage is varied. It is interesting to note that the maximum increase in overhead ia approximately $20 \%$ (for a link breakage rate of $50 \%$ ). Otherwise, for link breakages less than $10 \%$, the increase in overhead is approximately $10 \%$. One might argue that the figure is not insignificant. In fact, assessing whether this increase in overhead is acceptable or not really depends on the advantages brought out by multi-path routing. This is why a theoretical study such as the one proposed in the following is necessary.

\section{Simulation Results}

In the simulation, we choose Dynamic Source Routing (DSR) [12] and Multi-path Routing Protocol with Load Balance (MRP-LB) [15] as typical candidates for shortest path and multi-path routing protocols respectively. The choice of these routing protocols does not limit the applicability of this result into the others. In other words, the result which is derived above is applicable to other reactive routing algorithms such as AODV, TORA. However, the result is not suitable for proactive and hydrid routing protocols.

Clearly seen from Fig 5, MRP-LB exhibits higher overhead than DSR which once again confirms the correctness of our analytical model. 
TABLE II

SUMMARY OF PARAMETERS FOR TRAFFIC ANALYSIS

\begin{tabular}{|l|l|}
\hline$R$ & Radius of the disk \\
\hline$\delta$ & Node density \\
\hline$\lambda$ & Node-to-node transmission rate \\
\hline$\lambda_{m}$ & $\begin{array}{l}\text { Node-to-node transmission rate for multi-path } \\
\text { routing }\end{array}$ \\
\hline$\eta$ & Node processing rate \\
\hline$r$ & Distance of the node of interest to the disk center \\
\hline$L_{m}$ & Average length of a route in multi-path routing \\
\hline
\end{tabular}

IV. Traffic Analysis of Single Shortest Path AND Multi-PATH LOAD BALANCING ROUTING MECHANISMS

The following section compares the traffic distribution for the shortest-path and load-balancing routing mechanisms. We will be able to quantify the advantages in terms of congestion avoidance of the load-balancing routing mechanism over the shortest-path one. In particular, we will be able to determine in which networks multi-path routing really present interest. We will also derive an upper bound for a certain parameter which will guarantee that when multi-path routing mechanism is worth considering, it results in congestion decrease.

\section{A. Network Model}

In the model, we assume that mobile nodes are situated inside a disk with radius $R$. Furthermore, they are distributed uniformly with density $\delta$. In addition, mobile nodes communicate with each other at a uniform rate $\lambda$. Each node is assumed to have the same processing power of $\eta$ and have the same queue length of $Q_{\text {length }}$ for storing packets. The network model is shown in Fig 6. Clearly, we can see that the traffic going through each node consists of two types, i.e. the common traffic which is defined as a point-to-point communication traffic between nodes and the relay traffic which is defined as the forwarding traffic caused by data packets travelling through multiple hops to the destination. The parameters to be used in the analysis are summarized in Table II.

\section{B. Analysis of the Shortest Path Routing Algorithm}

It can be proved in Appendix on page 7 that the total traffic going through a node located at a distance $r$ from the center of the disk, $\lambda(r)$ can be expressed as follows:

$$
\lambda(r)=\left(\pi R^{2} \delta-1\right) \lambda+\frac{\pi\left(R^{2}-r^{2}\right)^{2} \delta^{2} \lambda \beta}{2}
$$

Therefore, according to Little theorem, the average number of packets in the queue for a node located at a distance $r$ from the center of the disk is:

$$
N_{p a c}(r)=\frac{\lambda(r)}{\eta-\lambda(r)}
$$

From the above equation, the total number of congested packet in the disk is:

$$
N_{\text {pactotal }}=\int_{0}^{R} 2 \pi r \delta N_{\text {pac }}(r) d r
$$

Hence, the average number of packets in a queue can be evaluated as:

$$
N_{p a c_{s}}=\frac{1}{\pi R^{2} \delta} * \int_{0}^{R} 2 \pi r \delta N_{p a c}(r) d r
$$

The exact calculation of $N_{p a c_{s}}$ is shown in the Appendix on Page 9. It is important for the following to know that $N_{p a c_{s}}$ can be exactly evaluated by integration and is a good indicator of the general congestion of the network.

\section{Analysis of the Multi-path Load Balancing Routing Mecha- nism}

A perfect load balancing multi-path routing mechanism distributes the traffic evenly among nodes in the network. As a consequence, "hot-spots" are eliminated. Therefore, packets are expected to experience lower average end-to-end delay. Suppose that $L_{m}, \lambda_{m}$ and $\eta$ are respectively the average length of a route in a network, the node to node traffic rate, and the processing rate. Let us evaluate the total traffic within the network. Since the number of nodes is $\pi R^{2} \delta$, it is easy to see that the total number of possible connections within the network is $\left(\pi R^{2} \delta-1\right) \pi R^{2} \delta$. With an average route length between two nodes of $L_{m}$ the total traffic within the network is $\left(\pi R^{2} \delta-1\right) \pi R^{2} \delta \lambda_{m} L_{m}$. Therefore, the incoming traffic per node is $\left(\pi R^{2} \delta-1\right) \lambda_{m} L_{m}$ and the average number of packets in the queue per node is:

$$
N_{p a c_{m}}=\frac{\left(\pi R^{2} \delta-1\right) \lambda_{m} L_{m}}{\left.\eta-\pi R^{2} \delta-1\right) \lambda_{m} L_{m}}
$$

In order to ensure that the load balancing policy decreases the congestion level of the network, $N_{p a c_{m}}$ should be smaller than $N_{\text {pac }}$. One can see in the above equation that the key parameter which controls $N_{\text {pac }_{m}}$ is the average length of a route. Indeed, in order to have $N_{p a c_{m}}<N_{p a c_{s}}, L_{m}$ must satisfy:

$$
L_{m}<\frac{N_{\text {pac }_{s}} \eta}{\left(N_{\text {pac }_{s}}+1\right)\left(\pi R^{2} \delta-1\right) \lambda_{m}}=L_{\max }
$$

This result shows that if $L_{m}>L_{\max }$, using a load balancing routing mechanism is no longer beneficial as compared with a shortest-path routing scheme. This can be easily implemented in practice: given a network characterized by its node density, its size and the traffic rate, one can evaluate $N_{\text {pac }_{s}}$. This value can then be used to calculate the theoretical value for $L_{\max }$ which is interesting because the result of this section can be used as a criteria to select the route in multi-path routing mechanism.

\section{Simulation Results}

Similarly to the previous section, DSR and MRP-LB are used to measure the traffic versus distance from the disk center. The results obtained from DSR and MRP-LB altogether with the result of ideal shortest path routing are shown in Fig 7. Clearly seen that, DSR demonstrates a consistent behavior to ideal shortest path routing in terms of traffic allocation. Clearly seen in the case of DSR, nodes closer to the disk center are experienced more traffic intensity, i.e. more congestion. However, in MRP-LB, due to the load balancing policy, mobile 
nodes are experienced approximately same traffic. Nevertheless, since MRP-LB is not an "ideal” load balancing protocol. Nodes closed to the disk center have to handle a slightly higher amount of traffic in comparison with ones at the rear.

In the next section, we will investigate another issue associated with a load balancing routing mechanism, namely the connection throughput of the network.

\section{Connection Throughrut Analysis}

In this section, we compare how the resources for transmission are used within the network for single-path and multi-path routing protocols.In order to conduct this study, we define the concept of connection throughput as follows:

Definition: The connection throughput of a network is defined as the average transmission rate of a connection in the network.

Note that the higher is the connection throughput, packets are experienced less delay during transmission. Therefore, the connection throughput is a good indicator of the average end to end delay in the network . Intuitively, we can see that congestion restricts the full usage of the available bandwidth. In other words, assuming that every route can support in theory a transmission at $W$ bits/seconds, the actual transmission rate of a route is limited by the fact that the bandwidth has to be shared with other routes at the MAC layer of each node. Therefore, the transmission rate of a route will be limited by the bandwidth available at the most congested node of this route. A load balancing policy which relieves "hot-spot" congestion should improve the connection throughput of the network. However, one has to be cautious since while the transmission rate in "hot-spot" areas increases due to congestion avoidance, it also decreases elsewhere in the network where more traffic is distributed. There is therefore a trade-off needed to consider when applying multipath routing mechanism. An interesting parameter characterizing the performance of multi-path routing is the average route length (calculated in number of hops). When this parameter increases, it results in more nodes in the network involved in connection, which means that more traffic is distributed across the network. In the followings, we propose an upper bound on the average length of a route in multi-path routing, which guarantees that the connection throughput is improved as compared to single-path routing.

\section{A. Single-path Routing}

In this section, we use the same network model as in section IV. According to (4), when a single-path routing mechanism is used, nodes closer to the disk center are experiencing more traffic, i.e. are more congested. Therefore, in terms of capacity, the total capacity of the network is limited by the capacity of the area close to the disk center. Consider a node A with distance $r$ from the disk center with radius $\mathrm{R}$ in Fig 8. Consider a connection between nodes $A_{1}$ and $A_{2}$. We will assume that the route between two nodes can be approximated by a straight line. We will later on discuss the limitation of this approximation. Let us denote by $A$, the orthogonal projection of the disk center $\mathrm{O}$ on the line $A_{1} A_{2}$. Assume that there is a node on the route between $A_{1}$ and $A_{2}$ very close to $A$. This is a valid assumption since we deal here with congested networks for which the node density is generally high. We will refer to this node as $A$ for the sake of simplicity. Since this particular node is closer to the disk center than any other nodes on the route, it experiences the highest traffic of route. Therefore the data transmission rate on this particular route is limited by the congestion experienced by node A. It can be easily seen from (4) that the number of routes going through node $A$ can be expressed as:

$$
n(r)=\left(\pi R^{2} \delta-1\right)+\frac{\pi\left(R^{2}-r^{2}\right)^{2} \delta^{2} \beta}{2}
$$

Assuming that we have a fair MAC layer, each route is allocated an equal bandwidth for data transmission. Therefore, each route going through node $A$ will be allocated the bandwidth denoted by $W(r)$ expressed as:

$$
W(r)=\frac{W}{\left(\pi R^{2} \delta-1\right)+\frac{\pi\left(R^{2}-r^{2}\right)^{2} \delta^{2} \beta}{2}}
$$

where $W$ is the total bandwidth allocated to the network. It can be recalled that $\pi R^{2} \delta=N$, the total number of nodes in the network. Assuming this number is large, then we assume that $\pi R^{2} \delta \approx N$.

Let us now evaluate the number of routes which transmission rate is limited by node $A$. Note that these routes have to be perpendicular to $O A$ and go through $A$. One can see in Fig 8 that these routes are such as their source and destination nodes are respectively in the areas $R_{1}$ and $R_{2}$ and vice versa. The number of nodes in each area can be expressed as:

$$
N_{R_{1}}(r)=N_{R_{2}}(r)=\left(R^{2}-r^{2}\right) \beta \delta
$$

The derivation which leads to this results is very similar to the one leading to (4). We will therefore refer our reader to Appendix on page 7 for more details. From this, the number of routes which transmission rates are limited by $W(r)$ is simply $2 N_{R_{1}}(r) N_{R_{2}}(r)$. Note that any node in the ring delimited by $r$ and $r+d r$ with $d r$ small enough will have the same traffic characteristics as $A(r)$. Therefore, it can be shown that $W_{s p}$, the total bandwidth used by the network will be expressed as:

$$
\begin{aligned}
W_{s p} & =\int_{0}^{R} W(r) 2 N_{R_{1}}(r) N_{R_{2}}(r) 2 \pi r \delta d r \\
& =2 W \delta \int_{0}^{R} \frac{\left(R^{2}-r^{2}\right)^{2} \beta^{2} \delta^{2}}{\left(\pi R^{2} \delta-1\right)+\frac{\pi\left(R^{2}-r^{2}\right)^{2} \delta^{2} \beta}{2}} 2 \pi r d r \\
& =2 W \sqrt{\frac{\beta N}{2 \pi}}\left(\sqrt{\frac{\beta N}{2 \pi}}-\arctan \sqrt{\frac{N \beta}{2 \pi}}\right)
\end{aligned}
$$

Note that we have used the fact $\mathrm{t}$ that $\pi R^{2} \delta=N$. The total number of possible connections being $N^{2}$, the connection throughput for this network using a single-path routing mechanism is $\lambda_{s p}=W_{s p} / N^{2}$

\section{B. Multi-path Load Balancing Routing}

Suppose that and $A_{c}$ is the average number of active routes per node. Obviously, the number of active routes in the network is $N A_{c} . L_{m}$ being the average number of nodes involved in a route, the total number of connections in the whole network is $N A_{c} L_{m}$ which means that the number of connections per node is $A_{c} L_{m}$. Assuming that the bandwidth available at each node is uniformly split among these connections, the bandwidth per per connection is $W /\left(A_{c} L_{m}\right)$. Therefore, the total bandwidth used by this network is:

$$
\begin{aligned}
W_{m p} & =\text { Number of active routes } \times \text { connection bandwidth } \\
& =N A_{c} W / A_{c} L_{m}=N W / L m
\end{aligned}
$$


The connection throughput is $\lambda_{m p}=W_{m p} / N^{2}$.

This result shows that the capacity of the network is inversely proportional to the length of a route. This confirms our initial comment that increasing the route length means distributing more traffic across the network, therefore decreasing the average connection throughput. It is therefore useful to compute an upper bound on $L_{m}$ which allows ensuring that

$$
\lambda_{m p}>\lambda_{s p}
$$

This leads to:

$$
L_{m}<L_{\max }^{\prime}=\frac{1}{2\left(\frac{\beta}{2 \pi}-\sqrt{\frac{\beta}{2 \pi N}} \arctan \left(\sqrt{\frac{\beta N}{2 \pi}}\right)\right)}
$$

It is worth noticing that $L_{\max }$ is itself bounded as follows:

$$
L_{\max }>\frac{\pi}{\beta}
$$

Remember that $\beta$ is a constant characterizing the fact that the routes between source and destination nodes are not perfect straight lines. This parameter, which only depends on the network density and node distribution, can be evaluated by simulations of geometrical analysis. When the network density is high, $\beta$ is typically small. Therefore, $L_{\max }$ will be a large number. For instance, for a network consisting of 100 nodes in 1 kilometer square, $\beta \approx \pi / 16$. We therefore have $L_{\max }>16$. However, on average, simulations show that the average path length in multi-path routing is around 6 or 7 hops. This means that there is in fact no constraint on $L_{m}$ as far as connection throughput improvement guarantee is concerned. In other words, using multi-path routing always improve the connection throughput of the network as compared to single-path routing. However, when the network density is low, $\beta$ is bigger, the value $L_{\max }$ must be taken into account as an upper bound of the routes when performing the route discovery so that a better performance is guaranteed when using multi-path routing.

\section{CONCLUSION}

In this paper, we have analyzed and compared single-path and multi-path routing algorithms. We have first concentrated this study on the issue of overheads. We have shown how the amount of overheads increases with the number of multiple paths and we have seen that when this number exceeds three, the overheads increase significantly. This has confirmed many simulations results presented in the literature which state without any clear explanation that using three paths provides the best trade off. We have also derived an upper bound on the average length of the multi-path routes which guarantees a decrease of the network congestion. This upper bound depends on the traffic intensity, the processing power of each node and the number of nodes in the network, hence is easy to compute in practice. Not only this bound allows to select routes that respect the upper bound constraint, but also, it can indicate in the first place whether for a particular network, using load balancing will bring any improvement at all. Finally, we have shown that using multi-path routing always results in connection throughput improvement for high density networks.

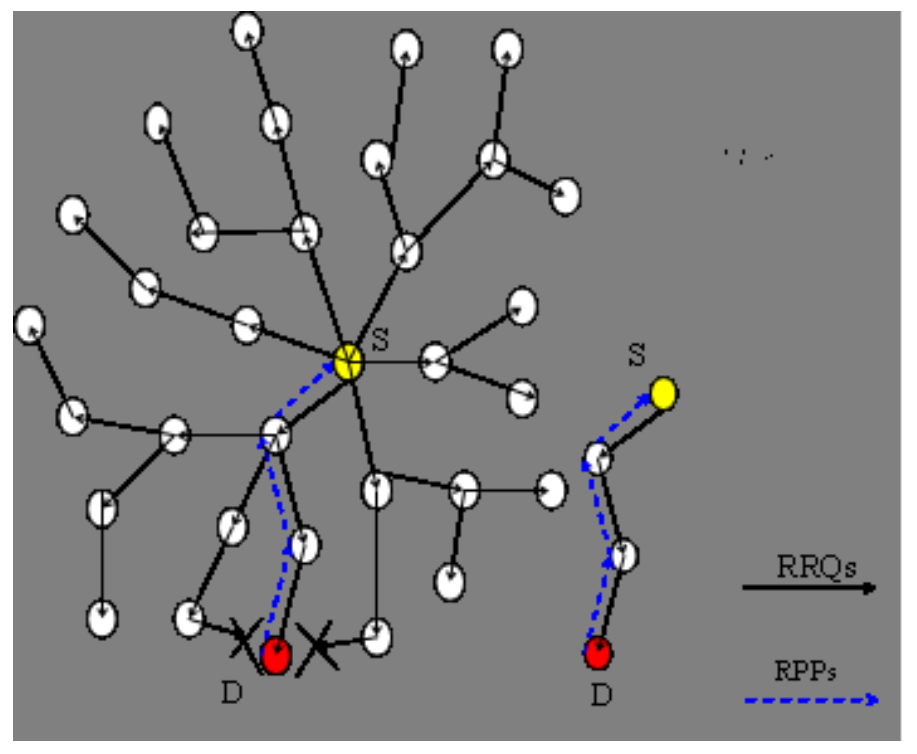

Fig. 1

Route Discovery in Single-Path Routing Mechanism

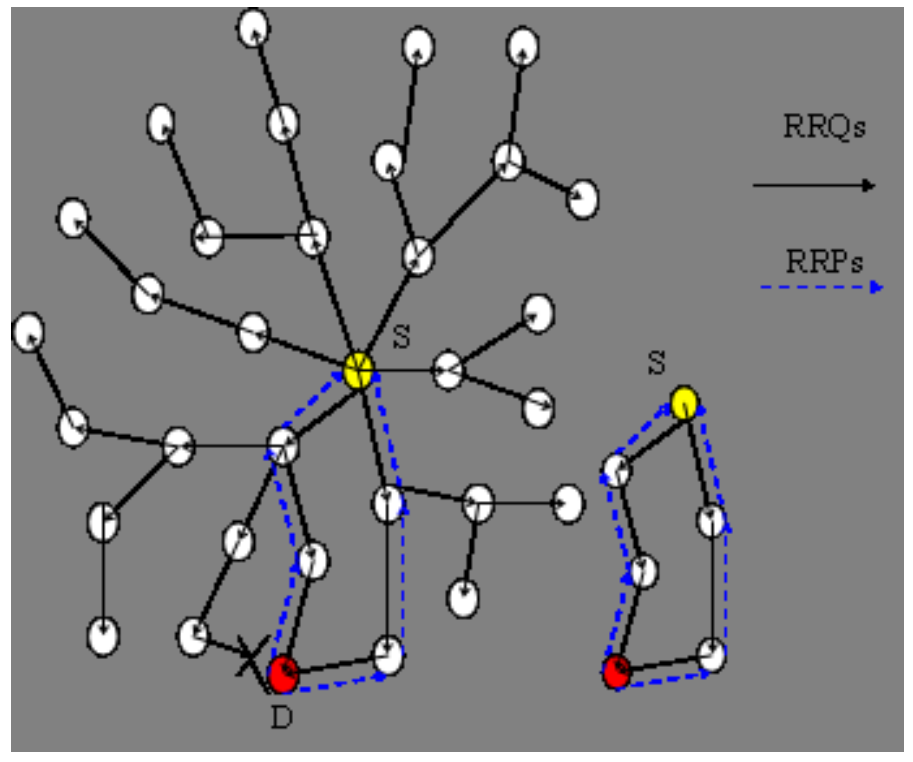

Fig. 2

Route Discovery in Multi-Path Routing

\section{REFERENCES}

[1] N.F. Maxemchuck, "Diversity routing," in IEEE ICC'75, San Francisco, CA, June 1975, IEEE, vol. 1, pp. 10-41.

[2] R. Krishan and J.A Silvester, "Choice of allocation granilarity in multipath source routing schemes," in IEEE INFOCOMM'93. IEEE, 1993, vol. 1, pp. 322-29.

[3] R.Rom I. Cidon and Y. Shavitt, "Analysis of multi-path routing," IEEE/ACM Transactions on Networking, vol. 7(6), pp. 885-896, 1999.

[4] R.C. Ogier and V. Ruthenburg, "Minimum-expected-delay alternate routing," in INFOCOMM, Florence, Italy, 6-May 1992, pp. 617-625.

[5] Nageswara S. V. Rao and S.G. Batsell, "Qos routing via multiple paths using bandwidth reservation," in INFOCOM (1), 1998, pp. 11-18.

[6] S.J. Lee and M. Gerla, "Aodv-br: Backup routing in ad hoc network," in IEEE WCNC 2000. IEEE, 2000, pp. 1311-16.

[7] L. Wang et al, "Multipath source routing in wireless ad hoc network," in Canadian Conf. Elec. Comp. Eng., 2000, vol. 1, pp. 479-83.

[8] S.J. Lee and M. Gerla, "Split multi-path routing with maximally disjoint paths in ad hoc networks," in ICC'01, 2001. 


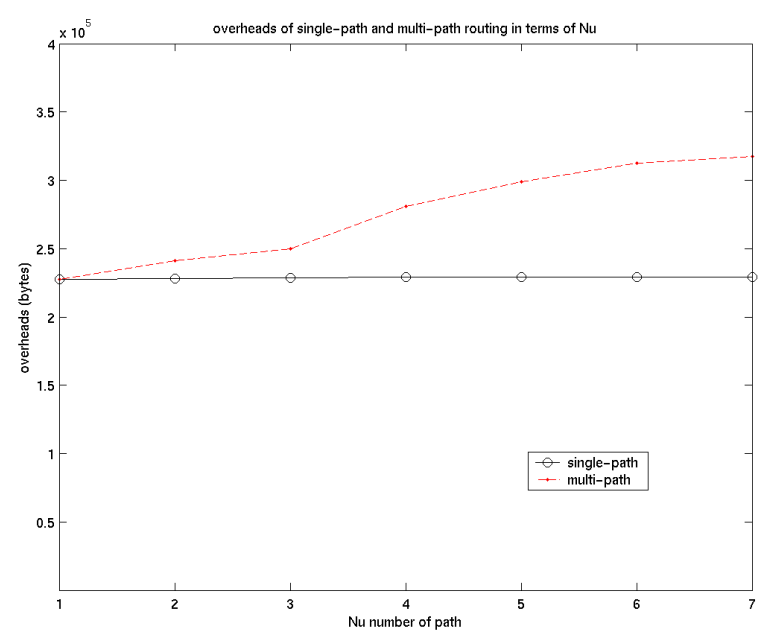

Fig. 3

OVERHEAD COMPARISON WHEN $N_{u}$ INCREASES

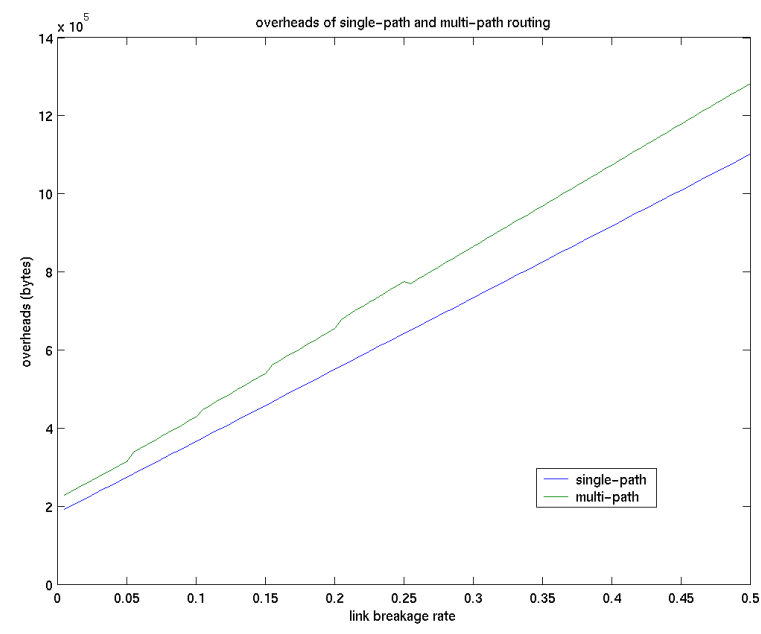

Fig. 4

OVERHEAD COMPARISON AS THE LINK BREAKAGE RATE INCREASES

[9] M.R. Pearlman et al, "On the impact of alternate path routing for load balancing in mobile ad hoc network," in MobiHOC, 2000, p. 150.

[10] A. Tsirigos and Z. J. Haas, "Multi-path routing in the presence of frequent topological changes," IEEE Communications Magazine, November 2001.

[11] A. Nasipuri and S.R. Das, "On-demand multi-path routing for mobile ad hoc networks," in IEEE ICCCN'99, 1999, pp. 64-70.

[12] D. Johnson and D. Maltz, "Dynamic souce routing in ad hoc wireless networks," in Mobile Comp..., T. Imielinkski and H. Korth, Eds. 1996, Kluwer.

[13] C. Perkins and E.M. Royer, "Ad-hoc on-demand distance vector routing," in IEEE Workshop on Mobile Computing Systems and Applications (WMCSA), 1999, pp. 90-100.

[14] V. D. Park and M. Scott Corson, "Temporally-ordered routing algorithm (tora) version 1: Functional specification.," Internet-Draft draft-ietfmanet-tora-spec-00.txt, November 1997.

[15] P. Pham and S. Perreau, "Multi-path routing protocol with load balancing policy in mobile ad hoc network," in IEEE MWCN'2002, 2002.

\section{APPENDIX}

Theorem 1: The traffic for a node located at a distance $r$ from the center of the disk can be expressed as represented by

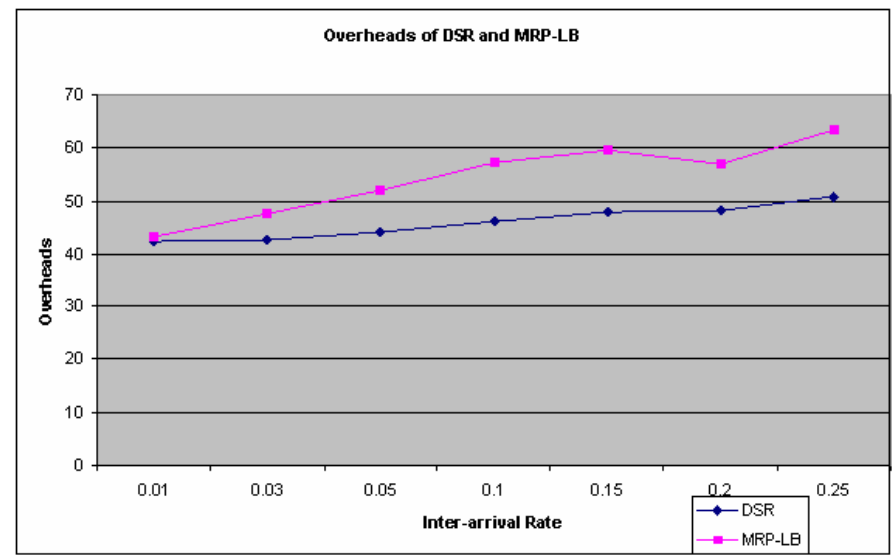

Fig. 5

OVERHEAD OF DSR AND MRP-LP

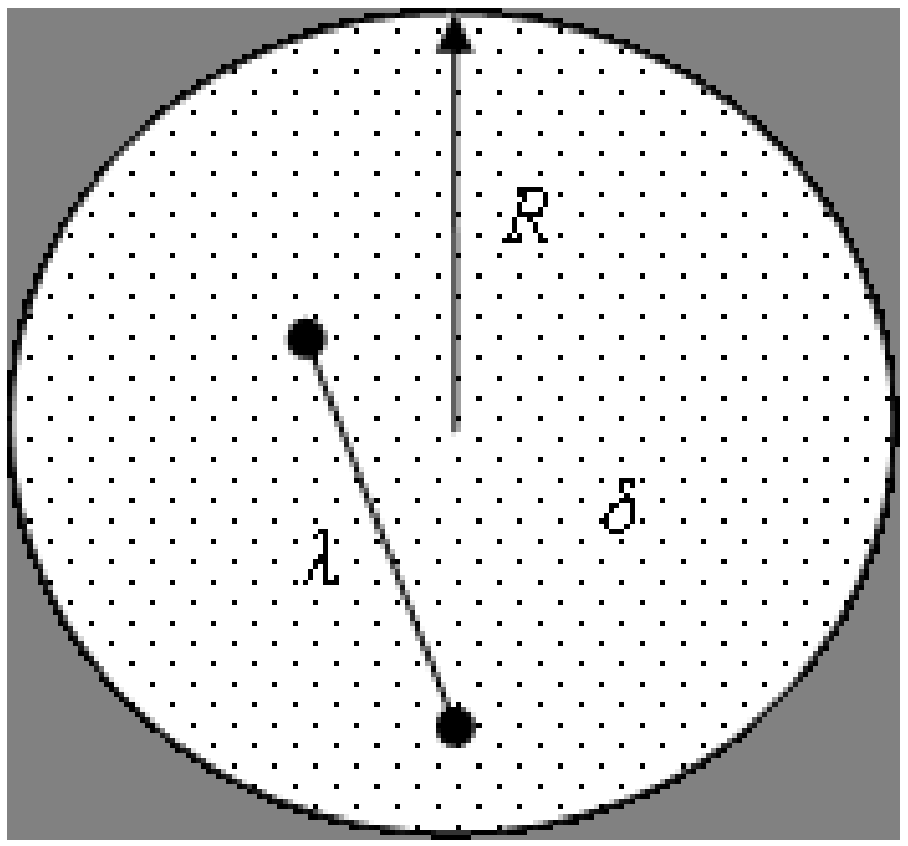

Fig. 6

NeTWork Model

the following expression:

$$
\lambda(r)=\left(\pi R^{2} \delta-1\right) \lambda+\frac{\pi\left(R^{2}-r^{2}\right)^{2} \delta^{2} \lambda \beta}{2}
$$

Proof: Let us denote by $A$, a node located at a distance $r$ from the center of the disk. Let us also define the following notation $x(i)$ is a point on the edge of the disk such as the angle between $A x(i)$ and the axis $O A$ is equal to $i$. Consider $S_{\alpha} d(\alpha)$, the portion of the disk (shadowed area on the Fig 9) center around $(A, x(\alpha))$ with a aperture of $d \alpha$. Our aim is to determine the amount of traffic originated by source nodes in $S_{\text {alpha }} d(\alpha)$ and going through node $A$. Recall that we use a shortest path routing mechanism in this section. It is reasonable to assume that in this case, routes are "close" to straight lines. The problem is then to determine the "destination area" $D$, i.e. 


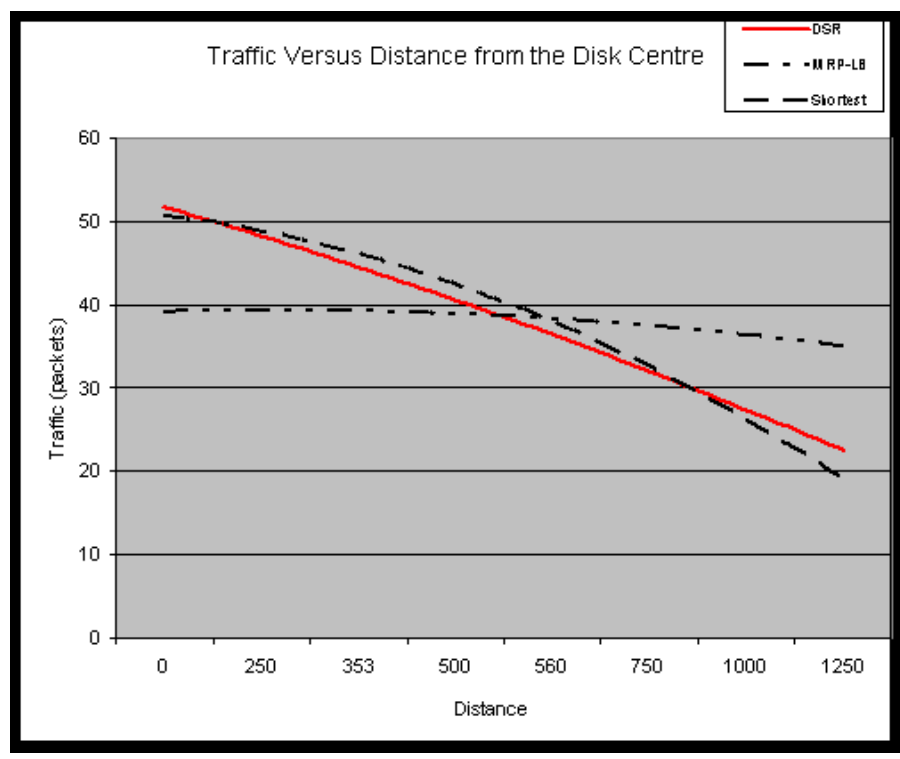

Fig. 7

TRAFFIC DISTRIBUTION OF DSR, MRP-LP AND IDEAL SHORTEST PATH

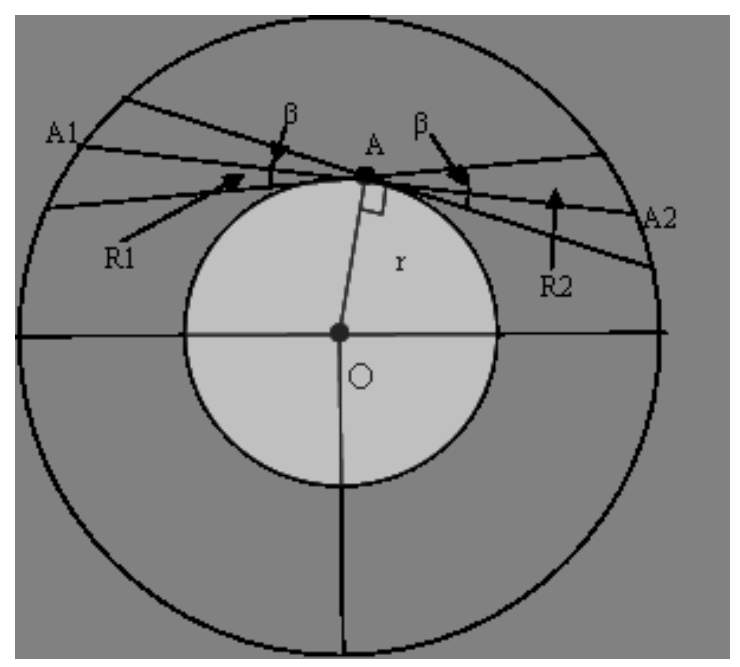

Fig. 8

NETWORK MODEL FOR CONNECTION THROUGHPUT

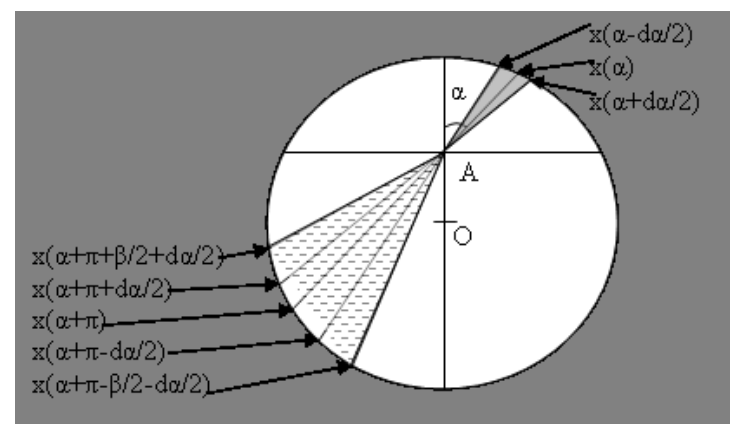

Fig. 9

TRAFFIC ANALYSIS FOR SHORTEST PATH MECHANISM

the portion of the disk containing all possible destination nodes corresponding with source nodes in $S_{\alpha} d(\alpha)$ through node $A$. If the routes were perfect straight lines, then obviously $D$ would be the portion of the disk (dashed area in Fig 9) centered around $(A, x(\alpha+\pi))$ with aperture $d \alpha$, i.e. $D=S_{\alpha+\pi} d(\alpha)$. However, since the routes are obviously not straight lines, D should be larger than this, i.e. $D=S_{\alpha+\pi}(d \alpha+\beta)$ with $\beta$ being a small positive real number, independent of $\alpha$ and $d \alpha$ is typically small. The value of $\beta$ is depending on network density and nodes distribution. This value can be obtained by using graphical analysis of nodes distribution or by simulation.

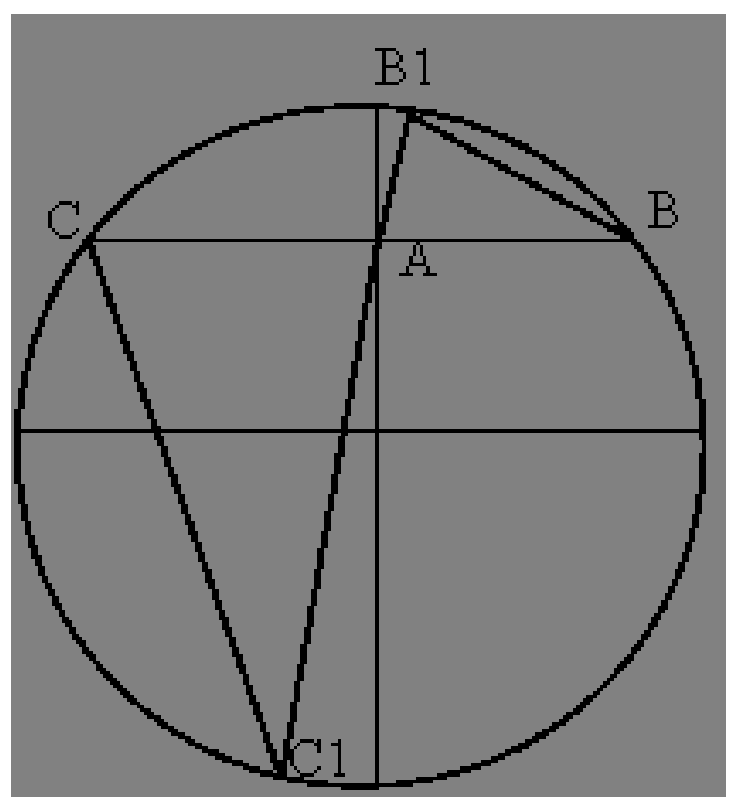

Fig. 10

ANALYSIS OF A LINE GOING THROUGH NODE A

Let us now evaluate $S_{\alpha} d(\alpha)$ and $S_{\alpha+\pi}(d \alpha+\beta)$. Since $d \alpha$ can be reasonably assumed small, the following approximations hold:

$$
\begin{aligned}
\sin (d(\alpha)) & =d(\alpha) \\
A x(\alpha-d(\alpha)) & =A x(\alpha) \\
A x(\alpha+d(\alpha)) & =A x(\alpha) \\
S_{\alpha} d(\alpha) & =\frac{A x(\alpha-d(\alpha)) \times A x(\alpha+d(\alpha)) \sin (d(\alpha))}{2}
\end{aligned}
$$

From these, we can conclude that

$$
S_{\alpha} d(\alpha)=\frac{A x(\alpha)^{2} d(\alpha)}{2}
$$

Similarly:

$$
S_{\alpha+\pi}(d \alpha+\beta)=\frac{A x(\alpha+\pi)^{2}(d \alpha+\beta)}{2}
$$

Assuming a uniform distribution of nodes in the dish then, the number of nodes in $S_{\alpha} d(\alpha) / 2$ and $S_{\alpha+\pi}(d \alpha+\beta)$ will be respectively $S_{\alpha} d(\alpha) \delta$ and $S_{\alpha+\pi}(d \alpha+\beta) \delta$ and therefore, the number of routes going through node $A$ will be:

$$
\begin{aligned}
N & =S_{\alpha} d(\alpha) \delta \times S_{\alpha+\pi}(d \alpha+\beta) \delta \\
& =\frac{A x(\alpha)^{2} d \alpha * A x(\alpha+\pi)^{2}(d \alpha+\beta) \delta^{2}\left(d \alpha^{2}+d \alpha \beta\right)}{4}
\end{aligned}
$$


With $d \alpha$ very small then $\left(d \alpha^{2}+d \alpha \beta\right)=\beta d \alpha$

Therefore:

$$
N=\frac{A x(\alpha)^{2} d \alpha A x(\alpha+\pi)^{2}(d \alpha+\beta) \delta^{2} \beta d \alpha}{4}
$$

In order to solve the problem, we have to prove the following result:

For any line $B 1 C 1$ going through node $A$, we have: $A C *$ $A B=A C 1 * A B 1=\left(R^{2}-r^{2}\right)$. From Fig 10, we can see that the triangle $\mathrm{AB} 1 \mathrm{~B}$ is similar to the triangle $\mathrm{ACC} 1$ because $\angle A B 1 B=\angle A C C 1$ and $\angle A B B 1=\angle A C 1 C$. Thus:

$$
\frac{A B 1}{A C}=\frac{A B}{A C 1}
$$

Therefore, $A B 1 \times A C 1=A B \times A C=R^{2}-r^{2}$. Since $A x(\alpha)$ and $A x(\alpha+\pi)$ are on the same straight line. It can be easily seen from (21) that:

$$
N=\frac{\pi\left(R^{2}-r^{2}\right)^{2} \delta^{2} \beta d \alpha}{4}
$$

Since traffic is bi-directional assuming that the traffic in both ways are the same, taking integration for $d \alpha$ from 0 to $\pi$, the relay traffic going through node $A$ will be:

$$
\text { relay-traffic }=\frac{\pi\left(R^{2}-r^{2}\right)^{2} \delta^{2} \beta \lambda}{2}
$$

The traffic incurs on each node includes the following traffic, traffic from others nodes and relay traffic. Since the circle is of radius $R$, the area $\pi R^{2}$. Therefore, the number of nodes in the circle are: $\pi R^{2} * \delta$. Hence, there are $\left(\pi R^{2} \delta-1\right)$ nodes communicating with the current node with traffic rate $\lambda$. The traffic on a node with distance $r$ from the center is:

$$
\begin{aligned}
\text { traffic } & =\text { common-traffic }+ \text { relay-traffic } \\
& =\left(\pi R^{2} \delta-1\right) \lambda+\frac{\pi\left(R^{2}-r^{2}\right)^{2} \delta^{2} \lambda \beta}{2}
\end{aligned}
$$

\section{Derivation of $N_{p a c_{s}}$}

From the derivation is Section IV.B, we have:

$$
N_{p a c}(r)=\frac{\lambda(r)}{\eta-\lambda(r)}=\frac{\left(\pi R^{2} \delta-1\right) \lambda+\frac{\pi\left(R^{2}-r^{2}\right)^{2} \delta^{2} \lambda \beta}{2}}{\eta-\left(\pi R^{2} \delta-1\right) \lambda-\frac{\pi\left(R^{2}-r^{2}\right)^{2} \delta^{2} \lambda \beta}{2}}
$$

and

$$
N_{p a c_{s}}=\frac{1}{\pi R^{2} \delta} * \int_{0}^{R} 2 \pi r \delta N_{p a c}(r) d r
$$

Therefore,

$$
\begin{aligned}
N_{\text {pac s }} & =\frac{1}{\pi R^{2} \delta} \int_{0}^{R} 2 \pi r \delta N_{p a c}(r) d r \\
& =\frac{1}{\pi R^{2} \delta} \int_{0}^{R} 2 \pi r \delta \frac{\left(\pi R^{2} \delta-1\right) \lambda+\frac{\pi\left(R^{2}-r^{2}\right)^{2} \delta^{2} \lambda \beta}{2}}{\eta-\left(\pi R^{2} \delta-1\right) \lambda+\frac{\pi\left(R^{2}-r^{2}\right)^{2} \delta^{2} \lambda \beta}{2}} d r \\
& =\frac{1}{\pi R^{2} \delta} \int_{0}^{R} \frac{2 K r\left[A+B\left(R^{2}-r^{2}\right)^{2}\right]}{\mu-A-B\left(R^{2}-r^{2}\right)^{2}} d r
\end{aligned}
$$

Where:

- $K=\pi \delta$

- $A=\left(\pi R^{2} \delta-1\right) \lambda$

- $B=\pi \delta^{2} \sin (\beta) \lambda / 2$

Hence,

$$
\begin{aligned}
N_{\text {pac s }_{s}} & =\frac{1}{\pi R^{2} \delta} \star \frac{K \mu / B}{2 \sqrt{\frac{\mu-A}{B}}} \ln \left(\frac{\sqrt{\frac{\mu-A}{B}}+R^{2}}{\sqrt{\frac{\mu-A}{B}}-R^{2}}\right)-\frac{1}{\pi R^{2} \delta} K R^{2} \\
& =\frac{\mu / B}{2 R^{2} \sqrt{\frac{\mu-A}{B}}} \ln \left(\frac{\sqrt{\frac{\mu-A}{B}}+R^{2}}{\sqrt{\frac{\mu-A}{B}}-R^{2}}\right)-1
\end{aligned}
$$

QED. 